\title{
A proposito di alcune mostre: "Art Life Politics" e il regime fascista
}

\section{di Adolfo Mignemi}

Ott 14, $2020 \mid \underline{\text { In evidenza, }}$ Storia pubblica $|\underline{0}|$

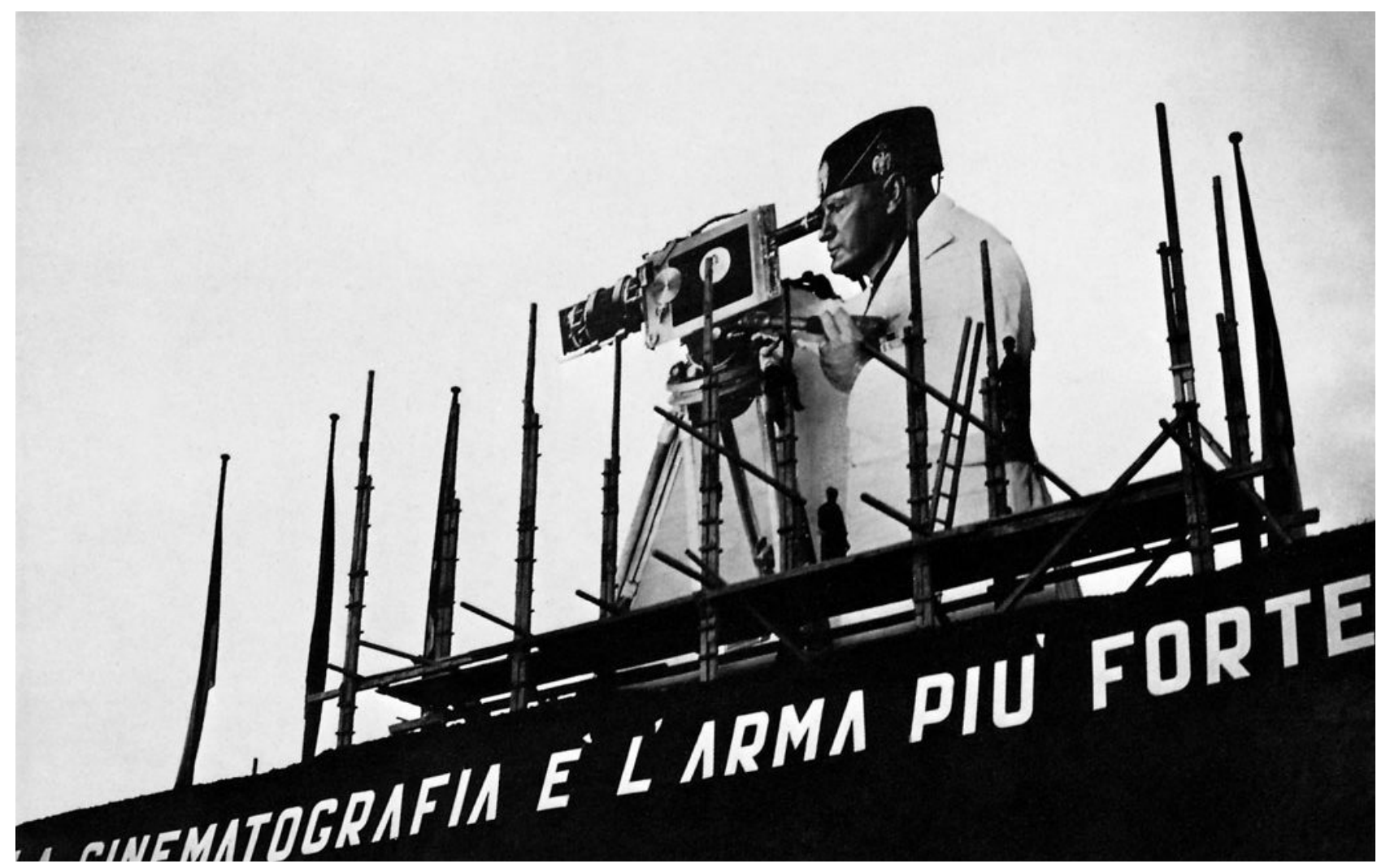

Apparato scenografico, con gigantografia di Mussolini alla macchina da presa e scritta propagandistica "La cinematografia è l'arma più forte", allestito per la cerimonia di fondazione della nuova sede dell'Istituto Luce (10/11/1937)

Foto di F.Romano10 - Own work, CC BY-SA 4.0, Link

\section{Abstract}

Pubblichiamo la seconda puntata della riflessione di Adolfo Mignemi che ripercorre alcune importanti mostre sulla storia italiana, e in particolare sull'epoca fascista, tenutesi nei mesi prima della chiusura forzata in seguito alle misure emergenziali anti-Covid 19.

Il testo fornisce utili strumenti per imparare a guardare i percorsi espositivi con occhi ben aperti, capaci di cogliere ciò che viene mostrato e, ancor più, quello che viene sottratto allo sguardo ma che, proprio per la sua assenza, produce senso.

\section{Rappresentare il ventennio fascista}

\section{L’ingorda parata di capolavori}

Se la mostra dedicata al premio Cremona si può definire come "la mostra della storia negata", 1'esposizione Post Zang Tumb Tuuum Art Life Politics Italia 1918-1943, promossa qualche mese 
prima a Milano dalla Fondazione Prada (peraltro citatissima da Sgarbi nel catalogo Il Regime dell'arte[1]) deve essere etichettata come "la mostra della elaborazione storica assente". In essa infatti si propone un numero impressionante di opere tutte realizzate nell'arco di tre decenni nel corso dei quali si sono succedute due guerre mondiali, si sono affermate forme politiche autoritarie espressione di ideologie che hanno condotto a ulteriori conflitti minori di violenza inaudita, il pianeta ha visto i suoi modelli economici di sviluppo precipitare in una crisi cupa e prodursi una contrapposizione ideologica e di dominio imperialistico che ne hanno riconfigurato gli assetti geopolitici. Nella mostra di tutto ciò sembra si sia fatto ampio impegno a non rendere minimamente conto, sospendendo in continuazione l'analisi storica dei contesti all'interno dei quali le opere erano nate e sostituendo a quella analisi una fitta quanto inutile cronologia, tutta concentrata in un lunghissimo testo che occupava un paio di muraglioni fittamente scritti. Ma poiché la domanda di storia non risponde ovviamente a una impellente necessità fisiologica, quando i visitatori raggiungevano le due mete, appagata sommariamente la curiosità e colta l'eterogeneità dei contenuti della proposta, rinunciavano inevitabilmente a leggere per rituffarsi nell'ordinata e lineare "ingorda parata di capolavori", come è stato definito polemicamente da qualche critico il percorso espositivo.[2]

\section{L’assenza di rielaborazione storica}

Ma vediamo come questa disinvolta operazione è stata raccontata e condotta nella realtà.

Il "progetto espositivo" della mostra è stato realizzato e presentato dal 18 febbraio al 25 giugno 2018 dalla Fondazione Prada nella propria sede milanese. La mostra - si poteva leggere nel comunicato stampa ufficiale - concepita e curata da Germano Celant, si prefiggeva il compito di esplorare

il sistema dell'arte e della cultura in Italia tra le due guerre mondiali, partendo dalla ricerca e dallo studio di documenti e fotografie storiche che rivelano il contesto spaziale, temporale, sociale e politico in cui le opere d'arte sono state create, messe in scena, nonché vissute e interpretate dal pubblico dell'epoca.[3]

E oltre:

Il periodo storico tra il 1918 e il 1943 è caratterizzato in Italia dalla crisi dello stato liberale e dall'affermazione del fascismo, nonché da una costante interdipendenza tra ricerca artistica, dinamiche sociali e attività politica. Come ha sottolineato Jacques Rancière nel suo libro "Le partage du sensible. Esthétique et politique" (2000), l'arte non esiste mai in astratto, ma si forma e prende forma in un determinato contesto storico e culturale. In questo senso l'aspetto politico e quello estetico sono inscindibili. Partendo da questo assunto, le testimonianze fotografiche e testuali che sono all'origine della selezione delle opere in mostra, documentano la produzione artistica e culturale del periodo tenendo conto di una pluralità di aspetti e ambienti in cui è realizzata ed esposta: dall'atelier d'artista alle collezioni private, dalle grandi manifestazioni pubbliche alle esposizioni e rassegne d'arte italiana in ambito nazionale e internazionale, dalle architetture ai piani urbanistici, dalla grafica alla prima produzione in serie di arredi. Secondo Germano Celant, $i$ documenti ritrovati e presentati oggi in questo progetto "sintetizzano la funzione comunicativa dell'opera d'arte, offrono una storia reale, fuori dalla trattazione teorica dell'artefatto". Funzionano come mezzi di "cultural understanding", per usare l'espressione di David Summers, che "garantiscono all'oggetto d'arte un territorio particolare, quello di apparire ad un'audience allargata, in determinate situazioni sociali e politiche”.[4] 


\section{L'uso delle fotografie}

Una prima osservazione, rilevante sul piano del metodo utilizzato da chi ha progettato la mostra: riguarda l'utilizzo della fotografia come documento. Osservava Germano Celant nel suo contributo teorico edito in catalogo: la fotografia cattura "la testimonianza della realtà del mostrare". E, aggiunge: "offre una percezione del luogo in cui la storia dell' arte è entrata in scena in modo ufficiale e non ufficiale, e permette un'interpretazione di manufatti insieme al loro contesto esistenziale".[5] Di qui la scelta guida di ricorrere sul piano del metodo al "rendering fotografico" nella costruzione dell'intero percorso espositivo.

Al tempo stesso però il visitatore si trovava di fronte a un "diverso" modo di operare visto che - un esempio per tutti di questo disinvolto uso documentale della fotografia - per richiamare la data simbolica della presa del potere ci si affidava a una fotografia acquerellata della marcia su Roma. Immagine colorata quasi sicuramente oggi in quanto quelle tonalità pastello sovrapposte al bianco e nero sarebbero apparse, allora e nell'arco di tutto il ventennio, assai poco consone a restituire la "vittoria" nel conflitto ideologico che si era consumato in quei mesi e, non ultimo, assai poco retoriche per celebrare un avvenimento che cadde, forse non a caso, nell'anniversario di un altro evento da secoli ritenuto epocale: la sconfitta di Massenzio da parte di Costantino nella battaglia di ponte Milvio nel 312 d.C.. Non si sottovaluti il fatto che quelle cronologie storiche erano ben radicate nella testa dell'ex maestro elementare Benedetto Mussolini, come chiunque può facilmente constatare anche semplicemente sfogliando gli inventari dei carteggi della sua Segreteria particolare, una volta diventato "il Duce".

L'inciso non è un'ampia divagazione ma volutamente si sofferma su una questione di fondo: la fotografia nella esperienza della comunicazione visiva del periodo fascista fu elemento e strumento di punta della elaborazione propagandistica, pertanto il ricorso ai caratteri documentali della fotografia non può mai prescindere da una attenta valutazione di tale contesto e di tutti i suoi potenziali nessi.

\section{Il fascismo "Pop"}

Riprendiamo dunque la lettura della presentazione ufficiale della mostra proposta da Prada:

L'indagine, svolta in collaborazione con archivi, fondazioni, musei, biblioteche e raccolte private, ha portato alla selezione di oltre 600 lavori, tra dipinti, sculture, disegni, fotografie, manifesti, arredi, progetti e modelli architettonici, realizzati da più di 100 autori. In "Post Zang Tumb Tuuum. Art Life Politics: Italia 1918-1943" questi oggetti sono introdotti da immagini storiche, pubblicazioni originali, lettere, riviste, rassegne stampa e foto personali per un totale di 800 documenti, così da mettere in discussione, come sostiene Germano Celant, "l'idealismo espositivo, dove le opere d'arte, nei musei e nelle istituzioni, sono messe in scena in una situazione anonima e monocroma, generalmente su una superficie bianca, per riproporle in relazione a una testimonianza fotografica d'epoca e nel loro spazio storico di comunicazione". Ricostruire, le condizioni materiali e fisiche della loro presentazione originale non solo consente di indagare il complesso sistema di relazioni tra autori, galleristi, critici, ideologi, politici, collezionisti, mecenati e spettatori, ma permette anche di esplorare il dispositivo di mostra nelle sue diverse declinazioni, come un elemento essenziale dell'universo simbolico del tempo. Una lettura che sottolinea ulteriormente come l'esposizione di immagini e di prodotti nazionali, anche in contesti 
internazionali, sia stata utilizzata dal fascismo come uno strumento flessibile, adattabile e moderno, un mezzo funzionale al progetto di rifare gli italiani e di plasmare la loro esperienza del mondo. Nella mostra "Post Zang Tumb Tuuum", l'artefatto, inserito nuovamente nel flusso caotico dell'esporre, ritorna a essere una materia viva, una costruzione stratificata di significati e possibili interpretazioni.

Il progetto di allestimento, ideato dallo studio $2 x 4$ di New York in dialogo con il curatore, si presenta come un percorso immersivo, ritmato da 24 ricostruzioni parziali di sale espositive pubbliche e private. In questi ambienti, costituiti dall'ingrandimento in scala reale delle immagini storiche, vengono ri-collocate le opere originali di artisti come Giacomo Balla, Carlo Carrà, Felice Casorati, Giorgio de Chirico, Fortunato Depero, Filippo de Pisis, Arturo Martini, Fausto Melotti, Giorgio Morandi, Scipione, Gino Severini, Mario Sironi, Arturo Tosi e Adolfo Wildt, tra gli altri. Si rinnova cosi l'osmosi tra espressione artistica e aspetti contestuali, come arredi, elementi architettonici, dettagli decorativi e soluzioni allestitive, che permette una conoscenza maggiore delle opere esposte e degli artisti e un'interpretazione più approfondita della storia delle arti in Italia. Si ripercorre cosi la dialettica tra singoli autori ed esponenti di movimenti, gruppi e tendenze, come Futurismo, Valori Plastici, Novecento, Scuola romana, i cosiddetti Italiens de Paris, il gruppo degli astrattisti e Corrente, che animano un panorama artistico e culturale, caratterizzato da eclettismo e pluralismo espressivi e in cui convivono avanguardia e ritorno all'ordine, sperimentazione e realismo, intimismo e propaganda. [6]

Come sempre tra il dire e il fare sta di mezzo il mare.

Le ambizioni della mostra apparivano molto grandi. "L'attenzione al contesto sociale, politico e vitale", si poteva leggere nella illustrazione ufficiale della esposizione si traduce

anche nella presentazione di progetti architettonici, piani urbanistici e allestimenti di grandi eventi quali la Mostra della Rivoluzione Fascista (1932), l'Esposizione dell'Aeronautica Italiana (1934), la Mostra nazionale dello Sport (1935) e l'imponente disegno dell'E42. Alcuni degli esiti più innovativi della concezione architettonica e scenica di questo periodo, come i contributi fondamentali del Gruppo 7, Giovanni Muzio, Marcello Piacentini, Piero Portaluppi e Giuseppe Terragni, tra gli altri, sono veicolati in mostra anche attraverso proiezioni di grandi dimensioni che permettono di restituire criticamente l'imponenza della scala originale e l'impatto comunicativo, propagandistico e celebrativo degli allestimenti dell'epoca, nonché di esplorare il processo di estetizzazione della politica e delle masse attuato dal fascismo.[7]

\section{Guarda un po' chi non c’è}

Peccato che, inoltrandosi in questo percorso, il visitatore non fosse adeguatamente accompagnato anche a ricordarsi degli eventi socio-politici che la società italiana aveva vissuto contestualmente in quegli anni così che anche il richiamo al "processo di estetizzazione della politica" veniva privato nella mostra dalla sostanza, ovvero di quel suo essere posto al servizio di una tragica spirale di avvenimenti che videro il "bel Paese" conquistarsi, prima in Africa e successivamente in Spagna, una fama odiosa e degna unicamente dell'abbraccio stabilito con il nazismo e l'imperialismo nazionalista nipponico, che porterà a scegliere di condividere con quelle due nazioni la responsabilità di trascinare il pianeta nel secondo conflitto mondiale.

Tra le lodevoli intenzioni della mostra, rientrava quella di aprire ampi squarci sull'intera vicenda del rapporto tra le varie arti, incluse quelle "minori", con il progetto politico del regime fascista e 
quindi con le sue scelte di comunicazione politica, sia con gli italiani sia con il resto del mondo. La scelta stessa del titolo della mostra, con quell'ammiccare al linguaggio del futurismo, doveva molto probabilmente preludere a tutto ciò, tanto che il visitatore accorto era legittimato e forse anche reso ansioso di incontrarsi, che so, con la declamazione radiofonica del Bombardamento di Adrianopoli, mandato in onda dall'Eiar nel 1929, o con la narrazione della Giornata dell'Ala a Roma, celebrata l'8 giugno dell'anno successivo mostrando al pubblico civile, nello spazio dell'aeroporto di Ciampino, il bombardamento di una raffineria di petrolio, il salvataggio da parte dei nuclei di paracadutisti di ostaggi trattenuti da un gruppo i ribelli libici e altre simili esercitazioni militari. Oppure, ancora, con le radiocronache da Ostia nel 1933 dell'arrivo dei trasvolatori atlantici guidati da Balbo.

Sono tutti contesti che ben si sarebbero coniugati con le coloratissime tele degli "Aeropittori futuristi" che interpretarono (e svilupparono, anche) il moderno concetto di militarizzazione del Paese propugnato dal regime. Opere, a loro volta, che si prestano a documentare la dialettica tra arte e politica così come si sviluppò in Italia.

Sarebbe stato impossibile inserire tutto ciò nella mostra, nessuno poteva pretenderlo. Di certo, però, sarebbero potuti essere citati schematicamente, collocandoli nel contesto, ad esempio, della italianissima elaborazione della dottrina di Giulio Douhet sulla guerra aerea e della parallela, intensa propaganda agitatoria in tali ambiti che percorse l'Europa nei primi anni Trenta.

\section{Che cosa è la propaganda?}

Si evocava prima anche l'aspettativa che la mostra sembrava voler creare verso il suono e lo sviluppo della radiofonia (così come per gli altri media emergenti: fotografia e cinema), ma l'Eiar nelle varie sale e nelle vetrine non c'era proprio, al di là di un manifesto di Nizzoli che annunciava nel 1927 l'inaugurazione in Roma della più potente stazione radiofonica d'Europa. Eppure gli ascolti collettivi nelle "grandi adunate" avevano egregiamente svolto il ruolo di dare físicità all'incontro con il Duce e allo sviluppo dl suo "culto" facendoli comprimari con la politica delle immagini e in particolare con l'uso dei cinegiornali Luce.

Della prima si è detto a più riprese soffermandosi sui limiti nell'utilizzo dei caratteri documentali delle immagini fotografiche. Circa il cinema la mostra aveva risolto il problema rinviando il visitatore ad un percorso specifico.

All'interno del Cinema della Fondazione Prada - illustrava il comunicato stampa generale - sono proiettati 29 cinegiornali integrali, selezionati in collaborazione con l'Istituto Luce - Cinecittà, distribuiti nelle sale italiane tra il 1929 e il 1941. I filmati documentano le fasi di allestimento e $i$ momenti di inaugurazione di alcuni tra i principali eventi espositivi e culturali del periodo.

In corrispondenza con la mostra sarà proposta inoltre una rassegna cinematografica che costruirà un dialogo tra film italiani realizzati tra il 1918 e il 1943 e opere cinematografiche dello stesso periodo prodotte in Germania, Unione Sovietica, Regno Unito, Francia, Giappone e Stati Uniti. [8]

Proposta di grande suggestione, peccato che essa finiva per non poter coinvolgere la stragrande maggioranza dei visitatori, in primo luogo quelli non provenienti direttamente da Milano. 
Nulla suppliva a queste non secondarie limitazioni, per quanto esse fossero relative ai due più innovativi mezzi di comunicazione di massa che vennero affermandosi in quegli anni in tutte le nazioni.

\section{L'estromissione della storia}

Abbiamo già sottolineato come fosse palesemente impossibile inserire tutto nell'esposizione, tuttavia, fornire un chiaro e solido quadro relativo alla comunicazione politica avrebbe giovato, eccome, a consentire al visitatore di entrare nel cuore delle opere che in essa venivano esposte.

Affermando ciò pensiamo, da un lato, anche a quella ampia rassegna di edizioni a stampa (a partire dalle invenzioni futuriste fino al "monumentalismo" vero e proprio dei volumi celebrativi) che avrebbe potuto e dovuto essere quanto meno inquadrata nella discussione su estetica e tecnica grafica (la cosiddetta "tipografia") che animò anche un interessante e intenso dibattito a metà degli anni Trenta (si pensi ai protagonisti: "Campo grafico" e "Il Risorgimento grafico"). Invece nulla, neppure un breve accenno sui muraglioni cronologici.

A giustificazione di questa omissione non regge neppure la scusa che il progetto generale della mostra era saldamente ancorato alla volontà di analizzare le esposizioni artistiche di quei decenni e pertanto che tutte le esposizioni al di fuori di questo ambito erano semplici ed estemporanei percorsi offerti alla golosità del pubblico. Le cose non stanno ovviamente così, visto che non possono essere ridotti a questo banale rango il richiamo agli allestimenti delle varie mostre "politiche", da quella della "Rivoluzione fascista" alla esposizione dell'Aeronautica, a quella dello Sport e, per finire, al progetto dell'E42. La prima e questa ultima godono nell'allestimento di spazi significativi.

Si rammenti, circa la prima, la suggestiva e coinvolgente restituzione delle sale del palazzo dell'Esposizione di via Nazionale a Roma, che la struttura della Fondazione Prada aveva realizzato nello spazio enorme del deposito. Anche in questo caso, se è lecito richiamare le possibili aspettative di un generico visitatore interessato a conoscere eventuali approfondimenti, sarebbe stato assai utile richiamare il carattere "permanente" della mostra organizzata per il decennale della marcia su Roma e delle sue successive versioni, fino a quella itinerante allestita nel 1942.

I materiali per farlo c'erano tutti: dal manifesto ufficiale, disegnato da Sironi, che in calce precisava "riduzioni ferroviarie 70\%" ai carnet di biglietti e di agevolazioni offerte ai visitatori con un modernissimo senso delle potenziali ricadute derivanti dalla organizzazione di un evento coordinato e "totalizzante" per chi aveva scelto di portare a termine questo "pellegrinaggio laico".

A questo proposito va anche aggiunto che la mostra della "Rivoluzione fascista" fu, a suo modo, per il regime terreno sperimentale nella caratterizzazione delle potenzialità rese disponibili dalla esposizione delle "prede di guerra" e delle "reliquie laiche". A Roma vennero infatti esposti moltissimi materiali di questo genere: dalle bandiere rosse "conquistate" nell'assalto alle Camere del lavoro o delle sedi socialiste, agli oggetti più disparati della "memoria storica militante", come la stampella appartenuta a Enrico Toti. Le guerre combattute in Africa, in Spagna e poi sui vari fronti europei divennero fonte di raccolte analoghe riproposte in pace e in guerra nelle innumerevoli "mostre" ed "esposizioni" allestite sotto l'egida ufficiale del fascismo.

Peccato non aver trovato minima traccia di tutto ciò nel percorso allestito alla Fondazione Prada. Esso avrebbe facilmente illuminato il nesso che pone in relazione la comunicazione politica e quelle semplificazioni che siamo soliti definire "propaganda". 
La constatazione viene a confermare la valutazione fin qui ripetutamente ribadita sul progetto generale della mostra: in essa dominava la voluta "utilità" offerta da una determinata e continua estromissione della storia nell'analisi dei materiali esposti.

\section{L'oblio "costruito" con la tecnica dei "braghettoni"}

Paradossalmente, ad accentuare questa propensione all'oblio contribuivano anzi i vari "focus tematici" dedicati - citiamo sempre dal comunicato stampa della Fondazione -

a figure di politici, intellettuali, scrittori e pensatori, come Pietro Maria Bardi, Giuseppe Bottai, Piero Gobetti, Antonio Gramsci, Carlo Levi, Alberto Moravia, Margherita Sarfatti e Lionello Venturi, in cui si analizzano le loro diverse posizioni in un momento di forte radicalizzazione delle idee, di scambio tra le arti e di dialogo o scontro aperto tra le persone. In questo clima l'intellettuale, cosi come l'artista, sviluppa la propria autonomia espressiva partecipando attivamente o restando indifferente alle indicazioni del regime, o al contrario, subendone o criticandone, in rari casi, le imposizioni in campo politico, culturale e artistico.

In mostra questa confusa miscela tra le elaborazioni di "fronda" interna all'esperienza fascista e le manifestazioni del pensiero alternativo, democratico e antifascista, che si manifesta nei venticinque anni considerati dalla mostra, muoveva in realtà dalla voluta costruzione di un equivoco di fondo: porre sullo stesso piano realtà non comparabili, antitetiche perché divise dalla durissima persecuzione subita dagli oppositori del regime. Si ricorreva infatti ai quadri di Carlo Levi, ai carteggi relativi all'allontanamento dall'Università di Lionello Venturi (uno dei dodici docenti che rifiutarono di prestare giuramento di fedeltà a Mussolini), alle fotografie di Gobetti, al volume dedicato a Casorati stampato nelle sue edizioni o alla sua rivista La rivoluzione liberale, a una lettera di Gramsci, all'immagine della gogna di Francesco Misiano per le vie di Roma, a qualche numero di Giustizia e Libertà (per dare forse concretezza all'evocazione del nome dei fratelli Rosselli) nella logica di creare un diversivo compensatorio creando uno spazio di apparente attenzione a tutto ciò che in Italia non era normalizzato dal fascismo.

La mostra allestita da Celant portava a compimento questo percorso ricorrendo - ci si passi il termine - a un espediente osceno: la "nobilitazione" dello status di intellettuale quasi che a questi livelli culturali ci debba essere sempre una sorta di reciproco rispetto. Ai promotori della mostra però non crediamo proprio fosse sconosciuto il fatto che Gobetti morì in seguito alle bastonature fasciste, che Gramsci fu lasciato marcire in carcere, che i fratelli Rosselli furono fatti assassinare in Francia da Mussolini. E così via. Un elenco davvero lungo e impressionante, assai difficile da ignorare.

Perché allora questo sconcio tentativo di minimizzare le distanze tra la cultura improntata al fascismo e quella della libertà e della democrazia di chi aveva scelto di opporsi con ogni mezzo al potere conquistato con la violenza squadrista? Siamo interamente immersi nella ipocrita ideologia che da sempre ha dato spazio ai "braghettoni" di turno e alla politica delle "foglie di fico", apposte alle cose che suscitano disprezzo da parte di chi la elabora e al tempo stesso morbose curiosità! 
Ma vorremmo sottolineare come anche in questo caso il carattere di una mostra, che si propone di fatto come quella della elaborazione storica assente, fornisca una ennesima conferma della strumentalità di questi suoi vizi d'origine.

Tre esempi. Il primo: la totale assenza dalla mostra dell'antifascismo di classe, quello nei confronti del quale operò principalmente, ma non solo, il Tribunale speciale per la difesa dello Stato, tra il 1926 e il 1943. In quell'arco di tempo esso emise 978 sentenze per reati politici, condannando 4596 imputati (il 98\% dei quali erano operai, artigiani e contadini mentre solo lo $0,4 \%$ era composto da professionisti), comminando 27.752 anni di carcere, tre ergastoli e 42 condanne a morte.

Il secondo esempio: la vicenda di Gramsci. Essa era evocata in mostra da una lettera scritta dal carcere di Turi il 17 giugno 1929. In essa Antonio scriveva al fratello Carlo a proposito di alcune questioni familiari, anche di carattere economico. Gli annunciava l'invio di alcuni pacchi di libri, contenenti 34 opere che, raccomandava, "devi conservarli per conto mio senza darli a nessuno o imprestarli. Non devono uscire di casa: io devo poterci contare in qualsiasi momento". La lettera era rimasta inedita fino al 1965 e sicuramente restituisce le dinamiche contraddittorie vissute da tutte le persone incarcerate e perseguitate dal fascismo, ma non a prima lettura. E non a caso un attento visitatore della mostra, che della stessa aveva finito per dare un giudizio comunque positivo, aveva annotato: 'Un po' inutile mostrare i libri appartenuti a Gramsci, fa più riflettere il famoso busto di Toscanini di Adolfo Wildt: strano che il Maestro non l'abbia ripudiato, carico com'è della retorica del Grand'Uomo".[9]

Il terzo esempio ci è stato suggerito dall'esposizione, già ricordata, di una fotografia relativa alla gogna pubblica subita il 13 giugno 1921 per le vie di Roma da Francesco Misiano, parlamentare, a opera di squadristi fascisti, tra i quali primeggiava Roberto Farinacci. Costretto ad abbandonare 1'Italia con la famiglia ancor prima della presa del potere da parte fascista, Misiano trovò rifugio in Urss ove nel 1924 fondò per conto del Soccorso Rosso internazionale una casa di produzione cinematografica, divenendo un protagonista di primo piano di quel settore, realizzando 160 film e 240 documentari nonché contribuendo a far conoscere al mondo intero la cinematografia di quel Paese.

Vi è infine un aspetto semplificatorio delle dinamiche storiche estremamente deleterio che si affaccia dietro a questa idea che il rispetto tra "veri intellettuali" supera le barriere ideologiche. Esso prelude a quel giustificazionismo diffuso, applicato anche alle cose peggiori, che induce a ritenere che siano stati solo i rozzi e gli ignoranti ad aver perseguitato i Gobetti, i Gramsci, e ad aver assassinato i Matteotti e i Rosselli. Così alla fine solo le "mani" sono veramente colpevoli e, di conseguenza, solo i poveri sono brutti, sporchi e .... fascisti!

\section{Un cammino palesemente modificato in corso d'opera e con molta fretta}

È singolare che il titolo della mostra ponesse come termine ad quem il 1943 ma, nei fatti, l'esposizione si prolungasse fino al 1945 proponendo nell'ultima sala, quasi un "fuori sacco" inviato a termini conclusi dell'allestimento, quei quadri di Maccari, Levi, Sassu e Treccani a richiamare le ultime "esposizioni" macabre allestite dal neofascismo scegliendo come luogo di messa in scena non più le gallerie d'arte o i padiglioni espositivi, ma le vie e le piazze d'Italia ove la resistenza armata combatté per il riscatto morale dell'intero Paese. L'allestimento di questa appendice vedeva anche l'esposizione di disegni di Corrado Cagli realizzati durante l'internamento nel campo di Buchenwald, posti accanto a foto dello stesso scattate dagli operatori Alleati dopo il loro arrivo sul luogo. In un vorticoso ballo tra gli orrori della guerra, il ritratto di Terragni in divisa, 
intento a disegnare bozzetti di vita militare sul fronte russo nel 1942, stava accanto ad alcuni scatti realizzati da operatori tedeschi durante il rastrellamento del Grappa nel 1944 e a Malga Zonta il 2 agosto 1944, alla foto dei partigiani uccisi a Milano in piazzale Loreto il 10 agosto 1944 e a quella dei cadaveri di Mussolini e dei gerarchi fascisti esposti nella stessa piazza il 29 aprile 1945, alle immagini dell'eccidio di Bassano del Grappa del 26 settembre 1944.

La "leggerezza" di questo pensiero progettuale sembrava voler trovare il suo punto fermo di arrivo in una gaffe finale: la riproposizione di un'ultima gigantografia fotografica di un allestimento che questa volta è la mostra di Palazzo Ducale a Genova allestita nel settembre 1945. La mostra era dedicata alla Resistenza e alla lotta di Liberazione. Celant era genovese e, con una scelta un po' campanilistica, deve aver pensato di poter firmare così l'appendice aggiunta alla mostra. Peccato che l'esposizione di Genova del 1945 rappresentata nella fotografia scelta ripropose i pannelli che erano stati realizzati per l'omonima esposizione allestita all'Arengario di Milano nel luglio 1945 da Mario De Micheli, Duilio Morosini, Gabriele Mucchi, Albe e Lica Steiner, Luigi Veronesi.

Un vecchio adagio, un tempo sempre ricorrente, ammoniva che quando ci si adopera per imprese importanti occorre agire con calma e riflessione poiché "la gatta frettolosa mette sempre al mondo gattini ciechi”. Con un po' più di attenzione e minor incontrollata sicurezza si sarebbe potuto precisare con una didascalia minima quanto si stava guardando, evitando così uno spiacevole $\mathrm{e}$ immotivato sgarbo per la città che stava ospitando la mostra della Fondazione Prada.

Gli allestitori avrebbero potuto accorgersene per tempo se solo avessero sfogliato proprio uno dei tanti, forse troppi, libri che nella stessa sala, di fronte proprio alla fotografia in questione, si trovavano a disposizione del pubblico in quella sorta di allestimento della "bibliografia della mostra" realizzata quantificando in termini fisici, nello stile un po' rozzo del mucchio di libri usati accumulato alla rinfusa sul selciato alla tradizionale "Fiera di Sinigaglia" ogni sabato mattina sulle sponde della Ripa di Porta Ticinese.

Fin qui le considerazioni sviluppate sui molteplici aspetti della mostra, ricorrendo alle impressioni annotate a caldo nel corso di un paio di visite accurate.

\section{Perché non si è adeguatamente discusso?}

Vorremmo a questo punto aggiungere alcune riflessioni generali.

La mostra aveva come oggetti privilegiati di riflessione le esposizioni, principalmente artistiche, che caratterizzarono il periodo 1918-1945. Ciascuna di quelle esposizioni corrispondeva a un particolare progetto che la mostra alla Fondazione Prada aveva il compito di dipanare completamente, ma al tempo stesso avrebbe dovuto contestualizzare ampiamente per poter cogliere le ambizioni e le finalità per cui ogni esposizione era stata messa a punto.

Ci siamo sforzati di evidenziare come la mostra non abbia voluto affrontare a pieno questo percorso e come l'affidarsi alla pura e semplice cronologia che restituisce in modo uniforme il fluire del tempo abbia, alla fine, impedito di operare sul piano storico quel riordino che implica analisi e pertanto individuazione delle problematiche a cui deve seguire necessariamente l'interpretazione attraverso l'analisi documentale.

Nella mostra i "pezzi" selezionati erano l'unico elemento parlante ma in un contesto di totale rinuncia all'interpretazione. È come se lo storico si fosse proposto di sostituire ad essa la semplice 
selezione dei documenti, senza neppure enunciare le ragioni della scelta né rendere evidenti le implicazioni storiche che ogni pezzo portava con sé.

È per questa ragione che, ad esempio, l'assenza dell'Africa pesa come un enorme macigno precipitato a ostruire il percorso. Il conflitto iniziato nel 1935 fu la messa a punto di un sistema propagandistico funzionale al manifestarsi delle nuove forme di dominio (l"'impero") perseguite dal regime.

Così è anche per le altre importanti assenze: da un lato la guerra di Spagna con il radicalizzarsi del confronto ideologico e con il "conflitto armato" che veniva di nuovo a consumarsi in casa, in Europa, anticipando senza mezzi termini la politica che di lì a poco sarebbe stata praticata dell'alleanza politica stipulata tra Germania, Italia e Giappone. Dall'altro, il dilagare delle politiche razziali che videro incrociarsi negli stessi anni, da est a ovest e viceversa, pratiche che diventeranno esperienza comune (deportazioni, stermini ecc.) che ancora oggi suscitano inquietanti oblii (si pensi al negazionismo praticato sia in Europa sia in Asia).

Non possiamo permetterci di costringere in questo modo la storia, anche ragionando semplicemente intorno ad una mostra d'arte.

\section{Un'occasione perduta}

La mostra Post Zang Tumb Tuuum. Art Life Politics: Italia 1918-1943 era

accompagnata da un volume scientifico di 696 pagine che include più di 1.000 illustrazioni, edito dalla Fondazione Prada. La pubblicazione include il saggio del curatore Germano Celant, 15 testi di studiosi, storici e critici d'arte e architettura come Ruth Ben-Ghiat, Francesca Billiani, Maristella Casciato, Daniela Fonti, Emilio Gentile, Romy Golan, Mario Isnenghi, Lucy Maulsby, Antonello Negri, Elena Pontiggia, Sileno Salvagnini, Jeffrey Schnapp, Francesco Spampinato, Marla Stone, Alessandra Tarquini e un'ampia sezione composta da 64 approfondimenti tematici redatti in occasione della mostra.[10]

A parte l'onerosità dell'opera che ha suscitato vari, numerosi e vivaci commenti, essa conferma la disorganicità del progetto della mostra. I testi sono infatti palesemente realizzati su invito ma senza il coinvolgimento degli autori nel progetto della mostra.

Di qui un'ultima considerazione intorno al dibattito sulla mostra. Questo in una certa misura c'è stato nel mondo degli storici e dei critici dell'arte, ma finendo per proporsi e apparire come un regolamento di conti tra addetti agli allestimenti.

Gli storici purtroppo si sono tenuti fuori da questo dibattito. Dico purtroppo in quanto la condivisione dei diversi saperi sviluppata in "corso d'opera" è assai più utile del confronto polemico a distanza. È come se - questo vale sia per la ricerca storica generale sia per quella nel campo delle arti - si prediligesse al crescere comune del sapere l'esercizio dell'autorappresentazione.

Così non va. Così ogni ricerca, ogni progetto scientifico finiscono per servire a nulla. Anche nell'ambito della costruzione della conoscenza storica. 
Note:

[1] S. Campagnolo, Introduzione, in Si faccia un articolo di fondo... 'Il Regime Fascista', Farinacci e il Ventennio a Cremona, Cremona, Biblioteca Statale di Cremona, 2018, p. 16.

[2] F. Giannini, Post Zang Tumb Tuuum, a Milano un paradigma nuovo ma una mostra ripetitiva, bulimica e pericolosa, in Finestre sull 'arte, magazine on line, 2 maggio 2018

(https://www.finestresullarte.info/867n_recensione-mostra-post-zang..., cons. 23 luglio 2019).

[3] Fondazione Prada. Milano Venezia, Comunicato stampa, s.i.d.

(https://www.fondazionepradaorg, cons. 11 aprile 2018).

[4] Fondazione Prada. Milano Venezia, Comunicato stampa, s.i.d.

(https://www.fondazionepradaorg, cons. 11 aprile 2018)..

[5] G. Celant, Toward a real and contextual history/Verso una storia reale e contestuale, in Post Zang Tumb Tuuum Art Life Politics Italia 1918-1943, Milano-Venezia, Fondazione Prada, 2019, pp. 32-33/553-554.

[6] Fondazione Prada. Milano Venezia, Comunicato stampa, s.i.d.

(https://www.fondazionepradaorg, cons. 11 aprile 2018)..

[7] Fondazione Prada. Milano Venezia, Comunicato stampa, s.i.d.

(https://www.fondazionepradaorg, cons. 11 aprile 2018)..

[8] Fondazione Prada. Milano Venezia, Comunicato stampa, s.i.d. (https://www.fondazionepradaorg, cons. 11 aprile 2018)..

[9] A. Saibene, Post Zang Tumb Tuuum Art Life Politics Italia 1918-1943, 18 feb. 2019

(htts://zero.eu/it/eventi/ 102989-post-zang-tumb-tuuum-prada.milano, cons. 9 sett. 2019).

[10] Fondazione Prada. Milano Venezia, Comunicato stampa, s.i.d.

(https://www.fondazionepradaorg, cons. 11 aprile 2018).. 